Rev. Int. Contam. Ambie. 34 (3) 383-394, 2018

DOI: 10.20937/RICA.2018.34.03.02

\title{
SUSTRATO BIOFÍSICO PARA AGRICULTURA PROTEGIDA Y URBANA A PARTIR DE COMPOST Y AGREGADOS PROVENIENTES DE LOS RESIDUOS SÓLIDOS URBANOS
}

\author{
Alfredo Ricardo PÉREZ FERNÁNDEZ ${ }^{1 *}$, Mariana RUIZ MORALES ${ }^{2}$, \\ María Odette LOBATO CALLEROS ${ }^{2}$, Eloy PÉREZ VALERA ${ }^{3}$ y Perla RODRÍGUEZ SALINAS ${ }^{1}$
}

${ }^{1}$ Universidad Tecnológica Fidel Velázquez. Emiliano Zapata, el Tráfico, Nicolás Romero, Estado de México, México, C. P. 54400

${ }^{2}$ Universidad Iberoamericana. Prolongación Paseo de la Reforma 880, Lomas de Santa Fe, Ciudad de México, México, C. P. 01219

${ }^{3}$ Biosistemas Sustentables, S. A., Promotora de Inversión de Capital Variable. Camino a Lanzarote, Los Tubos, Ejido de San José el Vidrio, Nicolás Romero, Estado de México, México, C. P. 54449

*Autor para correspondencia: utfv.ambiental@gmail.com

(Recibido abril 2017; aceptado octubre 2017)

Palabras clave: valoración de residuos, insumos agrícolas, bioinóculos, sustentabilidad

\section{RESUMEN}

Resolver integral y sustentablemente los problemas derivados de los residuos sólidos requiere de la valorización de sus subproductos. El presente trabajo muestra una alternativa al uso de compost obtenido del tratamiento de la fracción orgánica de los residuos sólidos urbanos, al mezclarla con poliestireno y tepetate para sustituir sustratos de uso agrícola y urbano. El estudio incluyó el análisis de cuestionarios de seguimiento a clientes de una empresa productora de compost quienes la mezclan con otros materiales para elaborar sustratos donde se cultivan hortalizas y plantas ornamentales. A partir de este análisis y el estado del arte sobre el tema, se mejoraron las mezclas recomendadas por productores, seleccionándose las que dieron mejores resultados y reformulándose para su comparación con sustratos comerciales para agricultura urbana y protegida mediante un diseño experimental de bloques completos al azar (DBCA). Las pruebas experimentales se realizaron en especímenes de rosas y jitomates sometidos a cuatro tratamientos diferentes. Se evaluaron las propiedades nutricionales del sustrato y la productividad bajo condiciones controladas en un invernadero. Los resultados muestran que los sustratos biofísicos propuestos presentan propiedades físicas, químicas y biológicas que los convierten en un sustituto viable para su empleo en jardinería urbana o en agricultura protegida. Estos hallazgos contribuyen a incrementar el uso de compost como base para el desarrollo de sustratos agrícolas, reduciendo el impacto ambiental asociado a la extracción de tierra de monte comúnmente empleada en jardinería, así como a promover la posibilidad de generar productos más asequibles con mejores resultados en productividad.

Key words: valuation of solid waste, farm inputs, bioinoculants, sustainability 


\begin{abstract}
In order to solve the solid waste disposal problems, subproducts need to be valuable. The present work shows an alternative to the use of compost obtained from the treatment of the organic fraction of municipal solid waste mixed with polystyrene and limestone to replace substrata for urban agriculture and agricultural use. The study included analysis of follow-up questionnaires answered by customers of a composting facility who mix it with other materials to produce substrata for cultivation of vegetables and ornamental plants. From this analysis, mixtures recommended by producers were tested, selecting those with the best results. Then, they were reformulated for comparison with commercial substrata used in urban and protected agriculture through an experimental design of randomized complete block design (RCBD). The experimental tests were performed on specimens of roses and tomatoes subjected to four different treatments. Nutritional properties of the substrate and productivity were evaluated under controlled conditions in a greenhouse. The results show that the proposed biophysical substratum presents physical, chemical, and biological properties that make it a viable substitute for use in urban gardening or protected agriculture. These findings contribute to increase the use of compost as a basis for the development of agricultural substrates, while reducing the environmental impact associated with the extraction of forest soil commonly used in gardening, as well as promoting the possibility of developing more affordable products with better productivity results.
\end{abstract}

\section{INTRODUCCIÓN}

En México el $63 \%$ del suelo presenta pérdida de nutrientes, materia orgánica y microorganismos benéficos provocado por la erosión (CONAFOR y UACh 2013). Estas pérdidas se ubican en el rango de US\$16.2 a US\$ 32.4/ha, mientras que el costo de reemplazo de los nutrientes perdidos asciende a US\$ 22.1/ha (Cotler et al. 2011).

Según datos de la SEMARNAT (2009), las actividades agrícolas son la principal causa de erosión del suelo en México, a lo que se suma el efecto negativo sobre el ambiente por el abuso de fertilizantes y plaguicidas. Por lo que se requieren prácticas agrícolas de menor impacto ambiental y que incrementen la productividad del suelo para garantizar la autosuficiencia alimentaria de una población creciente.

Esta situación ha detonado la búsqueda de alternativas de producción más eficientes y sustentables, donde la agricultura protegida representa ventajas tales como: alto rendimiento, certidumbre productiva y la programación de volúmenes de producción. Sin embargo su implementación demanda insumos específicos para crear condiciones óptimas para los cultivos que permitan al productor mejorar su ingreso (Moreno et al. 2011).

Por otra parte, los pequeños productores en las urbes mexicanas exploran mecanismos para producir alimentos, a través de huertos de traspatio, azoteas verdes y macetas; con objetivos que abarcan desde la subsistencia, mejora alimentaria, complemento de la renta familiar y el ocio (Urbano-López 2013). No obstante, los sustratos empleados en estos sistemas productivos tienen elevados costos económicos o ambientales pues requieren productos de importación como la turba o Sphagnum ("peat moss"), que además implica dependencia tecnológica. Mientras que la tierra de monte o tierra negra, conlleva impactos ambientales por extraerse del bosque (Ayala-Sierra y Valdez-Aguilar 2008).

De acuerdo con el anuario estadístico de la producción forestal, durante 2013 se extrajeron de manera legal $49953 \mathrm{t}$ de tierra negra, de las cuales el $85 \%$ fueron del Estado de México y de Morelos, a lo que debe sumarse la extracción ilegal con los correspondientes impactos ambientales (SEMARNAT 2013).

Derivado de la necesidad de materiales adecuados para cultivos en agricultura urbana y protegida, se ha propiciado el uso y búsqueda de materiales que puedan emplearse como sustratos, tales como: compost, estiércol, vermiculita, turba, fibra de coco, esquilmos agrícolas, arena, arcilla, perlita, pumita, etc. Sin embargo, estos materiales en ocasiones adolecen de controles de calidad, son costosos, o implican impacto ambiental. Por lo que es deseable que los sustitutos presenten homogeneidad, sean asequibles económicamente e impliquen menor impacto ambiental (Cruz-Crespo et al. 2013).

En la agricultura urbana y protegida, el sustrato es esencial para cultivar, pues además de que el volumen de una maceta, una cama agrícola o un acolchado son 
limitados, una vez establecidos ya no es fácil modificarlos. Por esta razón sus propiedades físicas, además de proporcionar adecuado soporte a la planta deben proveer condiciones estables de aireación, retención de humedad y drenaje que permitan subsanar deficiencias químicas y biológicas del cultivo mediante el riego para su óptimo crecimiento (Cabrera 1999).

Estos sistemas agrícolas emplean sistemas de riego por goteo subsuperficial porque alcanzan eficiencias de hasta $95 \%$ en la aplicación del agua y permiten dosificar nutrientes por fertirriego para subsanar carencias nutricionales. Por lo que los sustratos deben permitir un adecuado flujo de agua (Guevara-Escobar et al. 2005). De acuerdo con Cruz et al. (2010), para lograr propiedades físicas, químicas y biológicas adecuadas en los sustratos, deben combinarse materiales orgánicos e inorgánicos que proporcionen a los cultivos el soporte físico y nutrimental adecuado para producir a gran escala y con calidad suficiente.

El empleo de mezclas de compost y medios inertes en invernadero, arroja resultados favorables para el desarrollo de cultivos (Márquez-Hernández et al. 2006). Adicionalmente, Moreno et al. (2005) reportan que emplear compost inhibe patógenos del suelo y puede también restaurar suelos agrícolas degradados, además de reducir costos al sustituir parcialmente fertilizantes y plaguicidas (Olivares-Campos et al. 2012).

En México se generan diariamente 107100 t de residuos sólidos urbanos (RSU) dentro de las cuales, de acuerdo con Saldaña et al. (2013), existen subproductos que por sus particularidades pueden valorizarse. Tal es el caso del poliestireno que representa el $0.4 \%$ del total de RSU, que pudiera mezclarse con compost obtenido del tratamiento de la fracción orgánica para así crear sustratos agrícolas como alternativa de valorización de los RSU (INECC y SEMARNAT 2012).

Emplear residuos debidamente tratados para formular sustratos tiene valor ambiental al devolver al ciclo productivo los materiales desechados que al reciclarse reducen presión sobre recursos naturales que no resisten altos ritmos de explotación (Zapata et al. 2005). Además, ciertos residuos pueden emplearse como base para elaborar biofertilizantes y sustratos, incrementando la producción y reduciendo impactos ambientales (Díaz et al. 2015).

Usar compost como componente orgánico activo del sustrato aportará nutrientes y microrganismos, además de incrementar la retención de humedad y la capacidad de intercambio catiónico mejorando las propiedades, químicas y biológicas del sustrato (Parra-Delgado et al. 2014). Por otro lado, el poliestireno por su lenta degradación mantiene características físicas como densidad, permeabilidad y porosidad que junto con el tepetate da consistencia a la mezcla para soportar adecuadamente la planta cultivada (Lemaire et al. 2005).

La valorización de los subproductos recuperados de los RSU al emplearse como sustitutos del suelo en agricultura urbana y protegida, mitigaría los impactos negativos provocados al ambiente. Lo anterior, al disminuir la extracción de tierra negra, al reciclar los nutrientes contenidos en la fracción orgánica obtenidos del suelo de cultivo y al reducir la cantidad de residuos sólidos urbanos desechados (Montgomery 2007).

Con estos antecedentes, en el presente trabajo se evaluaron mezclas obtenidas del tratamiento de subproductos recuperados de los RSU complementadas con tepetate para elaborar un sustrato que sustituya a otros sustratos comerciales o a aquellos con un elevado impacto ambiental. Lo anterior, con el objetivo de evaluar si las mezclas propuestas pueden sustituir a sustratos empleados en agricultura urbana y protegida en la producción de plantas de rosa y jitomate.

\section{MATERIALES Y MÉTODOS}

\section{Ubicación del experimento}

Las pruebas se realizaron en San José el Vidrio, municipio de Nicolás Romero, Estado de México en las coordenadas $19^{\circ} 38^{\prime} 58.93^{\prime \prime} \mathrm{N}$; 99²0'45.73" W a una altitud de $2517 \mathrm{msnm}$ en un invernadero tipo cenital de estructura metálica cubierto con plástico blanco lechoso y malla antiáfidos.

Para recrear condiciones óptimas de productividad, una vez construido el invernadero, se trató con fungicidas y plaguicidas para eliminar afectaciones. El riego se efectuó tres veces por semana con agua de la localidad tomada del suministro público, siguiendo recomendaciones de la literatura (Ortega-Martínez et al. 2014).

\section{Diseño del sustrato}

De acuerdo con Vence (2008), las propiedades físicas de los sustratos son las más importantes al estudiar materiales usados para cultivo en contenedores. Por lo que la finalidad de mezclar materiales es obtener un sustrato de suficiente consistencia para soportar a la planta así como con la porosidad necesaria para proporcionar una adecuada disponibilidad de agua y aire en las raíces.

El diseño del sustrato parte de la metodología de despliegue de función de calidad, en donde se traducen las necesidades y expectativas de los consumidores de un producto a un conjunto de especificaciones 
de diseño o manufactura (Gutiérrez et al. 2014). Esta información se obtuvo del análisis de datos recopilados de 2011 a 2013 por una empresa productora de compost, que da seguimiento de satisfacción a sus clientes a través de cuestionarios.

La información analizada corresponde a 530 productores de plantas de ornato y hortalizas en invernadero de los estados de México, Morelos y Querétaro. Quienes proporcionaron datos relacionados con área cultivada, especie sembrada, sustratos empleados y rendimientos alcanzados al emplear compost obtenido del tratamiento de la fracción orgánica de los RSU.

Para elaborar los sustratos se seleccionaron los materiales más productivos reportados por los productores así como los citados en bibliografía para producción en invernadero (García et al. 2001, Acosta-Duran et al. 2008, Cruz-Crespo et al. 2013).

Una vez identificados los materiales, se contempló que se mencionaran en los lineamientos establecidos en la descripción, especificaciones y declaraciones obligatorias y opcionales establecidas para el compost del anexo I del Real Decreto 865/2010 del 2 de julio sobre sustratos de cultivo (BOE 2010).

Mientras que para las proporciones de materiales a emplear, se siguieron las recomendaciones para mezclas de sustratos para producción en sistema tradicional y a raíz desnuda de la NMX-AA-170-SCFI-2014 (SE 2014). De esta depuración de información se realizaron 18 mezclas con 6 repeticiones cada una, empleando los materiales reportados: tezontle, arena, vermiculita, poliestireno, tierra negra, tierra de hoja, agrolita, turba, compost y tepetate.

Para evaluar los sustratos se empleó la rosa hibrida (Rosa sp.) por ser la especie ornamental más producida en el país con el $24 \%$ de la producción total y el jitomate saladette (Lycopersicum esculentum) que es la hortaliza de mayor participación en el mercado con $21.4 \%$ (SAGARPA 2015).

La variable de respuesta seleccionada para el jitomate saladette fue la producción en $\mathrm{kg}$ de fruto de jitomate evaluado durante todo el ciclo de producción. Para la especie ornamental, la variable de respuesta evaluada fue el número de botones de rosa producidos durante el ciclo fenológico.

\section{Diseño experimental}

Los sustratos fueron evaluados en dos etapas, en la primera se probaron los materiales reportados por los productores, cultivándose en ellos las especies de prueba para obtener los valores de las variables de respuesta. Las mezclas fueron preparadas con base en volumen (v:v) para constituir los tratamientos a evaluar mediante la comparación de resultados con otros sustratos empleados en agricultura urbana y protegida.

Después de obtenidos los resultados, se realizó una segunda corrida experimental empleando los materiales de mejor respuesta, para lo cual se prepararon las mezclas que constituyeron los tratamientos a comparar con sustratos comúnmente empleados en agricultura urbana y protegida.

En la primera etapa se cultivaron el jitomate saladette y la rosa en 18 mezclas con 6 repeticiones cada una, de los materiales que los productores encuestados reportaron como más productivos: tezontle, arena, vermiculita, poliestireno, tierra negra, tierra de hoja, agrolita, turba, compost y tepetate.

Los sustratos para jitomate se evaluaron cultivándolos en camas acolchadas con película de polietileno de alta densidad negro plata calibre 90, trasplantando la plántula producida en invernadero a 40 días de sembrada, separadas $20 \mathrm{~cm}$ entre matas con un volumen de sustrato de $20 \mathrm{~L}$. Como variable de respuesta se empleó la producción en $\mathrm{kg}$ de fruto de jitomate evaluado durante todo el ciclo de producción, siendo este de julio de 2014 a enero de 2015.

La evaluación de los sustratos para las rosas se realizó plantándolas en contenedores de polietileno lavados y desinfectados, con un volumen de $20 \mathrm{~L}$ de las mezclas, colocando una planta podada por contenedor. La variable de respuesta evaluada fue el número de botones de rosa producidos durante el ciclo fenológico de marzo de 2014, a junio de 2014.

Al finalizar el ciclo fenológico de las especies de prueba se cuantificaron las variables de respuesta de cada mezcla, seleccionándose los dos preparados con mejores resultados, siendo estos los constituidos a base de composta, tepetate y poliestireno. A estos sustratos se les determinó la distribución del tamaño de partículas para obtener la textura mediante el hidrómetro de Bouyoucos (Martínez y López 2001), pues el tamaño de partícula determina el resto de las propiedades físicas del suelo (Vargas-Tapia et al. 2008).

Para la segunda etapa se formularon los sustratos que constituirían los tratamientos a evaluar, los cuales por su distribución de tamaño de partícula, corresponden a un suelo de textura franca. La mezclas fueron preparadas empleando compost para proporcionar nutrientes combinado con tepetate y poliestireno para mantener la estructura que soporte a la planta así como la porosidad para permitir el flujo de aire y agua (Dorner y Dec 2007).

El primer tratamiento (T1) fue sustrato natural constituido al $100 \%$ por tierra de monte o negra adquirida en un vivero local. El segundo tratamiento 
(T2) fue sustrato comercial, compuesto por: $40 \%$ turba $+20 \%$ agrolita $+20 \%$ vermiculita $+20 \%$ humus. El tercer tratamiento (T3) se formuló con $34 \%$ compost $+33 \%$ tepetate $+33 \%$ poliestireno y el cuarto tratamiento (T4) con $50 \%$ compost $+25 \%$ tepetate $+25 \%$ poliestireno. (Cuadro I).

\begin{tabular}{|c|c|}
\hline & $\begin{array}{l}\text { LUMEN DE LOS SUSTRATOS EVALUADOS } \\
\text { PARA PRODUCCIÓN DE ROSA Y JITOMATE }\end{array}$ \\
\hline Tratamiento & Composición en volumen \\
\hline $\mathrm{T} 1$ & $100 \%$ tierra negra \\
\hline $\mathrm{T} 2$ & $\begin{array}{l}20 \% \text { agrolita }+20 \% \text { vermiculita }+20 \% \\
\text { humus }+40 \% \text { peat moss }\end{array}$ \\
\hline $\mathrm{T} 3$ & $\begin{array}{l}34 \% \text { composta }+33 \% \text { poliestireno }+33 \% \\
\text { tepetate }\end{array}$ \\
\hline T4 & $\begin{array}{l}50 \% \text { composta }+25 \% \text { poliestireno }+25 \% \\
\text { tepetate }\end{array}$ \\
\hline
\end{tabular}

El tepetate empleado contiene $64 \%$ de arcilla (Acevedo-Sandoval et al. 2003) y se extrajo de una excavación a $1.0 \mathrm{~m}$ de profundidad del sitio donde se ubica el experimento. El poliestireno fue recuperado de la fracción inorgánica de RSU, se trituró y se emplearon los materiales del cernido por separado que pasaron por una malla de $2.0 \mathrm{~mm}$.

El compost fue obtenido mediante tratamiento mecánico biológico de residuos orgánicos por el método de pila con volteo establecido en la norma NTEA-006-SMA-RS-2006 (SMA 2006). Una vez estabilizado el compost, se inoculó con $5.0 \mathrm{~g} / \mathrm{kg}$ del bioestimulante comercial Natur-Abono ${ }^{\text {BSS }}$ de la marca Biosistemas Sustentables, quienes garantizan al menos 30 esporas/g de hongos micorrízicos arbusculares de la especie Glomus intraradices.

La inoculación se realizó para establecer una asociación sustrato-hongo-raíz ecológicamente mutualista, para incrementar el aprovechamiento de nutrientes en las especies de prueba. Cuenca et al. (2007) reportan que estas inoculaciones favorecen la absorción de elementos poco móviles como fósforo $(\mathrm{P})$, cobre $(\mathrm{Cu})$ y zinc $(\mathrm{Zn})$; protección contra patógenos, resistencia a la sequía y contribuyen a la formación de la estructura del suelo. Otra importante ventaja es la fijación de nitrógeno $(\mathrm{N})$ pues los compost tienden a bajos niveles de $\mathrm{N}$ respecto a lo considerado como adecuado (Raj y Antil 2011).

Esta segunda etapa se realizó también en camas acolchadas para los jitomates con $20 \mathrm{~L}$ de sustrato por planta y la variable de respuesta fue la producción acumulada en kg durante todo el ciclo productivo evaluado mediante cortes semanales, siendo el primero el 21 de julio de 2015 y el último el 10 de febrero de 2016.

La evaluación de los tratamientos sobre las rosas, se realizó plantándolas en contenedores de $20 \mathrm{~L}$, estableciendo el número de botones como variable de respuesta, monitoreada semanalmente desde su aparición el 24 de marzo de 2015, hasta su corte el 10 de junio de 2015.

El efecto de los tratamientos sobre la variable de respuesta se obtiene hasta la culminación del ciclo fenológico de las especies de prueba, por lo que debe bloquearse el efecto de otros factores que pudieran incidir pero que no se consideran en el estudio. Para lograr este bloqueo, los tratamientos se dispusieron en un diseño experimental de bloques completos al azar (DBCA) con diez repeticiones, pues esta disposición es la recomendada en este tipo de experimentación agrícola (Ruiz-Ramírez 2010).

Al concluir el ciclo fenológico de las plantas, las variables de respuesta se evaluaron mediante un análisis de varianza de un factor y la comparación de medias se realizó mediante la prueba de Tukey ( $p$ $\leq 0.05$ ) en el programa estadístico Minitab 17 para establecer diferencias significativas debidas a los tratamientos empleados.

\section{Análisis químicos de los sustratos}

Para establecer si los sustratos biofísicos propuestos contienen elementos disponibles para la raíz que contribuyan o inhiban el desarrollo de las plantas, se realizaron análisis de los sustratos para determinar cualitativa y cuantitativamente los iones evaluados (Alcántar-González et al. 2016).

El método de análisis empleado para determinar las propiedades químicas de los sustratos fue por extracto de pasta saturado (EPS), metodología usada por laboratorios universitarios y comerciales de suelos; pues sus guías generales de interpretación están basadas en numerosos estudios y ensayos de fertilidad (Ansorena-Miner 1994). El EPS es un buen indicador de la disponibilidad de nutrientes y fitotóxicos en sustratos biofísicos. Se obtiene filtrando una solución del sustrato que ha sido saturado con agua desionizada para disolver los nutrientes primarios y secundarios; mientras que los micronutrientes son extraídos con ácido dietilentriaminopentaacético (DTPA).

Las determinaciones se realizan tomando una alícuota del sustrato y se mide $\mathrm{pH}$ potenciométricamente, nitratos $\left(\mathrm{NO}_{3}{ }^{-}\right)$por espectrofotometría uv, fosfatos $\left(\mathrm{PO}_{4}^{-}\right)$por el método de Morgan, sulfatos 
CUADRO II. pH Y CONTENIDO DE NUTRIENTES EN LOS SUSTRATOS EVALUADOS

\begin{tabular}{|c|c|c|c|c|c|}
\hline Parámetros físicos & $\mathrm{T} 1$ & $\mathrm{~T} 2$ & $\mathrm{~T} 3$ & $\mathrm{~T} 4$ & $\begin{array}{c}\text { Rangos óptimos de } \\
\text { suficiencia nutrimental }\end{array}$ \\
\hline $\mathrm{pH}$ & 7.10 & 6.10 & 7.90 & 7.70 & 5.6 a 7.8 \\
\hline NUTRIENTES PRIMARIOS & $(\mathrm{ppm})$ & $(\mathrm{ppm})$ & $(\mathrm{ppm})$ & (ppm) & (ppm) \\
\hline Nitratos & 304.0 & 806.0 & 16.0 & 24.0 & $21-40$ \\
\hline Fosfatos & 0.11 & 2.90 & 0.31 & 0.46 & $20-30$ \\
\hline Potasio & 52.0 & 356.0 & 337.0 & 577.0 & $487-731$ \\
\hline \multicolumn{6}{|l|}{ NUTRIENTES SECUNDARIOS } \\
\hline Calcio & 70.0 & 420.0 & 310.0 & 350.0 & $200-400$ \\
\hline Magnesio & 10.0 & 210.0 & 115.0 & 105.0 & $750-1500$ \\
\hline Sulfatos & 77.0 & 1249.0 & 1153.0 & 1441.0 & $144-960$ \\
\hline \multicolumn{6}{|l|}{ MICROELEMENTOS } \\
\hline Boro & 0.04 & 2.33 & 5.9 & 8.0 & $0.7-1.5$ \\
\hline Cobre & 3.4 & 5.2 & 26.2 & 35.5 & $0.4-0.8$ \\
\hline Zinc & 9.8 & 51.2 & 159.0 & 227.0 & $1.0-2.0$ \\
\hline Manganeso & 11.3 & 108.0 & 19.7 & 20.7 & $1.5-3.0$ \\
\hline Fierro & 81.0 & 88.0 & 104.0 & 149.0 & $3.0-6.0$ \\
\hline
\end{tabular}

$\mathrm{T}=$ tratamiento $($ Cuadro $\mathbf{I})$

*Fuente: Elaboración propia a partir de Alcántar-González et al. (2016)

$\left(\mathrm{SO}_{4}{ }^{2-}\right)$ por turbidimetría, carbonatos $\left(\mathrm{CO}_{3}{ }^{2-}\right)$ y bicarbonatos $\left(\mathrm{HCO}_{3}{ }^{-}\right)$por titulación. Por el método de Mohr cloruros $\left(\mathrm{Cl}^{-}\right)$, sodio $(\mathrm{Na})$, potasio $(\mathrm{K})$, calcio (Ca) y magnesio $(\mathrm{Mg})$ por absorción atómica. Los microelementos cobre $(\mathrm{Cu})$, zinc $(\mathrm{Zn})$, manganeso $(\mathrm{Mn})$ y fierro $(\mathrm{Fe})$ se extraen con DTPA y se cuantifican por espectrofotometría. El boro (B) se extrae con agua a ebullición y se determina por colorimetría con azometina (Rodríguez-Fuentes y Rodríguez-Absi 2002).

La interpretación agronómica se realizó comparando los resultados obtenidos contra niveles de referencia establecidos para diagnóstico agrícola de agua, suelo y material vegetal según Alcántar-González et al. (2016). Se muestran los valores encontrados en los cuadros II y III.

\section{Análisis foliar de las plantas}

Para explicar las diferencias sobre las variables de respuesta de los tratamientos propuestos, se determinan nutrientes primarios, secundarios y micronutrientes en las hojas, pues estos órganos son los que mejor reflejan el estado nutricional (Mujica Pérez 2012). Determinar la condición nutrimental del cultivo permite establecer si el sustrato biofísico provee lo necesario para el desarrollo de las especies de prueba; así como el tipo y cantidad de complementos minerales a adicionar para mejorar la calidad nutricional de los sustratos propuestos.

Los análisis se realizan preparando una muestra compuesta a partir de todas las hojas recolectadas de las plantas sujetas a cada tratamiento cuando estas alcanzan al menos el $20 \%$ de floración. La muestra

CUADRO III. CONTENIDO DE ANIONES EN LOS SUSTRATOS EVALUADOS

\begin{tabular}{lccccc}
\hline \multirow{2}{*}{ Iones } & T1 & T2 & T3 & T4 & $\begin{array}{c}\text { Rangos satisfactorios } \\
\text { para aniones* }\end{array}$ \\
\cline { 2 - 6 } & $\mathrm{Meq} / \mathrm{L}$ & $\mathrm{Meq} / \mathrm{L}$ & $\mathrm{Meq} / \mathrm{L}$ & $\mathrm{Meq} / \mathrm{L}$ & $\mathrm{Meq} / \mathrm{L}$ \\
\hline Carbonatos & 0.0 & 0.0 & 0.0 & 0.0 & 0.0 \\
Bicarbonatos & 0.60 & 2.4 & 16.0 & 20.0 & $0.1-2.5$ \\
Cloruros & 1.8 & 5.6 & 48.0 & 53.0 & $<5.0$ \\
Sodio & 2.7 & 3.1 & 54.4 & 62.6 & $<25$ \\
\hline
\end{tabular}

$\mathrm{T}=$ tratamiento (cuadro $\mathbf{I})$

*Fuente: Elaboración propia a partir de Alcántar-González et al. (2016) 
se seca a la sombra y se envía para su análisis a un laboratorio especializado.

La determinación de nitrógeno $(\mathrm{N})$ total se realizó mediante el método de Kjeldhal, el P por digestión con ácido sulfúrico y colorimetría (Paneque-Pérez et al. 2010). El Na, K, Ca, Mg, Fe, Zn, Cu y Mn mediante digestión ácida de la muestra y posteriormente por absorción atómica (Rodríguez-Fuentes y Rodríguez-Absi 2002), y para el boro (B), se calcina el tejido vegetal y se determina por colorimetría con azometina (McKean 1993).

Los datos del contenido de nutrientes foliares fueron comparados con el rango de suficiencia establecido para diferentes cultivos (Alcántar-González et al. 2016). Los resultados de este análisis se presentan en el cuadro IV.

\section{Análisis estadístico}

El análisis estadístico se realizó mediante un análisis de varianza de un factor de las variables de respuesta por tratamiento empleando la prueba de Tukey $(\mathrm{p} \leq 0.05)$ para evaluar diferencias significativas de las medias de producción de los tratamientos. Los análisis se hicieron a través del programa Minitab 17.

\section{RESULTADOS Y DISCUSIÓN}

Al finalizar el ciclo fenológico de las especies evaluadas, se observó que las mezclas empleadas cumplieron con los requerimientos para producir jitomate saladette (Lycopersicum esculentum) y rosa híbrida (Rosa sp.), lo que implica que los sustratos

CUADRO IV. ANÁLISIS FOLIAR DE LAS PLANTAS DE ROSAS SOMETIDAS A TRATAMIENTOS

\begin{tabular}{lrrrrc}
\hline & $\mathrm{T} 1$ & $\mathrm{~T} 2$ & $\mathrm{~T} 3$ & $\mathrm{~T} 4$ & $\begin{array}{c}\text { Rangos de } \\
\text { suficiencia nutrimental* }\end{array}$ \\
\cline { 2 - 6 } NUTRIENTES PRIMARIOS & $\mathrm{ppm}$ & $\mathrm{ppm}$ & $\mathrm{ppm}$ & $\mathrm{ppm}$ & $\mathrm{ppm}$ \\
\cline { 2 - 5 } Nitrógeno & 720 & 760 & 800 & 1000 & $28000-45000$ \\
Fósforo & 800 & 3480 & 1080 & 1040 & $2500-5000$ \\
Potasio & 16000 & 19000 & 25000 & 19000 & $18000-30000$ \\
NUTRIENTES SECUNDARIOS & & & & & \\
Calcio & 17000 & 21500 & 13800 & 12100 & $10000-15000$ \\
Magnesio & 2400 & 3700 & 2800 & 2400 & $3000-6000$ \\
Azufre & 1160 & 1820 & 1720 & 1271 & $2100-5000$ \\
MICROELEMENTOS & & & & & \\
Fierro & 93 & 89 & 81 & 95 & $80-150$ \\
Zinc & 32 & 44 & 43 & 47 & $25-80$ \\
Cobre & 6 & 1 & 1 & 1 & $7-15$ \\
Manganeso & 30 & 470 & 430 & 400 & $35-120$ \\
Boro & 35 & 80 & 40 & 30 & $30-70$ \\
\hline
\end{tabular}

$\mathrm{T}=$ tratamiento $($ cuadro $\mathbf{I})$

*Fuente: Elaboración propia a partir de Alcántar-González et al. (2016)

\section{CUADRO V. EFECTOS DE LOS TRATAMIENTOS SOBRE LAS VARIABLES DE RESPUESTA EVALUADAS}

\begin{tabular}{llrc}
\hline Tratamiento & Composición & $\begin{array}{c}\text { Medias de botones } \\
\text { por planta* }\end{array}$ & $\begin{array}{c}\text { Medias de producción } \\
\text { por jitomate }(\mathrm{gr}) *\end{array}$ \\
\hline T1 & $100 \%$ tierra negra & $2.7 \mathrm{a}$ & $10319 \mathrm{a}$ \\
T2 & $20 \%$ agrolita $+20 \%$ vermiculita $+20 \%$ humus $+40 \%$ peatmoss & $2.8 \mathrm{~b}$ & $9674 \mathrm{a}$ \\
T3 & $34 \%$ composta $+33 \%$ poliestireno $+33 \%$ tepetate & $2.0 \mathrm{a}$ & $10586 \mathrm{a}$ \\
T4 & $50 \%$ composta $+25 \%$ poliestireno $+25 \%$ tepetate & $1.8 \mathrm{a}$ & $12657 \mathrm{~b}$ \\
\hline
\end{tabular}

*Las letras distintas dentro de la misma columna para medias de variable de respuesta, denotan diferencias estadísticas significativas según prueba de Tukey $(\mathrm{p} \leq 0.05)$. 
propuestos pueden sustituir a los sustratos testigo empleados en agricultura urbana y protegida.

El efecto de los tratamientos sobre las variables de respuesta en la producción de botones de rosa y en $\mathrm{kg}$ de jitomate se puede observar en el cuadro $\mathbf{V}$.

Los valores para la variable de respuesta de la rosas cultivadas en maceta, muestran que los tratamientos T3 y T4 constituidos por composta, poliestireno y tepetate; no presentan diferencia estadística significativa respecto al número de botones producidos en $\mathrm{T} 1$ constituido al $100 \%$ por tierra negra. Mientras que el cultivo T2 constituido por: $20 \%$ agrolita $+20 \%$ vermiculita $+20 \%$ humus $+40 \%$ turba, sí presenta una media de producción de botones con diferencia significativa en contra de los tratamientos T3 y T4.

Para el cultivo de jitomate saladette en invernadero, el T4 formulado con $50 \%$ composta $+25 \%$ poliestireno $+25 \%$ tepetate, resultó con diferencia estadística significativa respecto a los otros tratamientos produciendo más fruto que los tres tratamientos restantes.

Las mezclas propuestas mantuvieron durante la prueba la consistencia y estabilidad de sus agregados en concordancia con lo reportado por Ghosh et al. (2010), quienes muestran que la adición de compost al suelo incrementa la estabilidad estructural del mismo.

Durante el experimento no se observaron cambios en el volumen de los sustratos que comprometieran el adecuado soporte físico para el desarrollo de la raíz, además de que suministraron los nutrientes requeridos en niveles aceptables para el desarrollo de las especies evaluadas (Cuadro II).

Con respecto al $\mathrm{pH}$, los cuatro sustratos biofísicos evaluados presentan valores que permiten la asimilación de nutrientes, por lo que no se esperan efectos por acidez o alcalinidad. Aunque los tratamientos T3 y T4, posiblemente debido al compost, caen en la categoría de medianamente alcalinos de acuerdo con la norma NOM-021-SEMARNAT (SEMARNAT 2001).

Los niveles de macronutrientes de los sustratos formulados con subproductos recuperados de los RSU presentan menos $\mathrm{N}$ mineral que la tierra negra y el sustrato comercial, pero contienen más $\mathrm{K}$ que los sustratos testigo, posiblemente aportado por el compost. Mientras que el contenido de $\mathrm{P}$ es bajo en los cuatro sustratos. Estos valores de macronutrientes ubican al tratamiento T3 por debajo del nivel óptimo y al T4 dentro del rango óptimo como puede observarse en el cuadro II.

Los sustratos a base de poliestireno, tepetate y compost aportan suficiente $\mathrm{Ca}$, semejante al proporcionado por el sustrato comercial (más de 300 ppm); no así la tierra negra, que con 70 ppm se coloca debajo de los niveles de suficiencia. Para los $\mathrm{SO}_{4}{ }^{2-}$, los sustratos con poliestireno y el comercial muestran niveles de suficiencia, mientras que la tierra negra solo tuvo $77 \mathrm{ppm}$. Por otro lado, de acuerdo con los valores de referencia, los cuatro sustratos son deficientes en $\mathrm{Mg}$, siendo la tierra negra la más carente de este nutriente.

Con excepción del B que fue bajo para la tierra negra, los otros micronutrientes estuvieron por encima de los niveles de suficiencia en los cuatro sustratos.

En el análisis de extracto saturado se determinan una serie de aniones que de ser excesivos incrementan la salinidad pudiendo ser fitotóxicos. El cuadro III muestra los valores encontrados en las mezclas propuestas de los iones que incrementan el riesgo de salinidad, que aunque son superiores a los sustratos testigo y a los considerados como rangos aceptables, no se evidenciaron afectaciones en las variables de respuesta de las especies de prueba.

En ninguna mezcla se encontraron $\mathrm{CO}_{3}{ }^{-}$. Pero los sustratos con poliestireno, composta y tepetate tuvieron cantidades altas de $\mathrm{HCO}_{3}^{-} \mathrm{Cl}^{-}$y $\mathrm{Na}$, indicando salinidad que disminuye los potenciales osmótico e hídrico del sustrato, lo que compromete la absorción de agua de las raíces de las plantas produciendo estrés hídrico (Bárcenas et al. 2002).

Los tratamientos propuestos permitieron el desarrollo de las rosas a pesar del exceso o defecto de nutrientes y de la presencia de iones con potencial de salinización como puede observarse en los resultados de los análisis foliares mostrados en el cuadro IV.

Esta comparación del efecto de nutrientes y iones sobre la variable de respuesta para las plantas de rosa se muestra en la figura 1 donde puede observarse que la tierra negra y el sustrato comercial obtuvieron más botones que los sustratos con poliestireno, compost y tepetate. Por lo que estas mezclas requerirán ajuste del nivel de nutrientes por las carencias mostradas en el cuadro II.

Para los jitomates, la productividad acumulada evaluada como variable de respuesta durante todo el ciclo productivo medido del 21 de julio de 2015 al 10 de febrero de 2016, muestra que los valores más altos se obtuvieron para el T4 formulado con compost, poliestireno y tepetate (Fig. 2).

La mezcla T3 arroja una media de producción por planta de $10.586 \mathrm{~kg}$, que es $2.6 \%$ más que el tratamiento con tierra negra y $9.4 \%$ más que el sustrato comercial; mientras que el T4 arroja una media de producción de $12.657 \mathrm{~kg}$, que es $22.7 \%$ más que el tratamiento de tierra negra y $30.8 \%$ más que el sustrato comercial. 


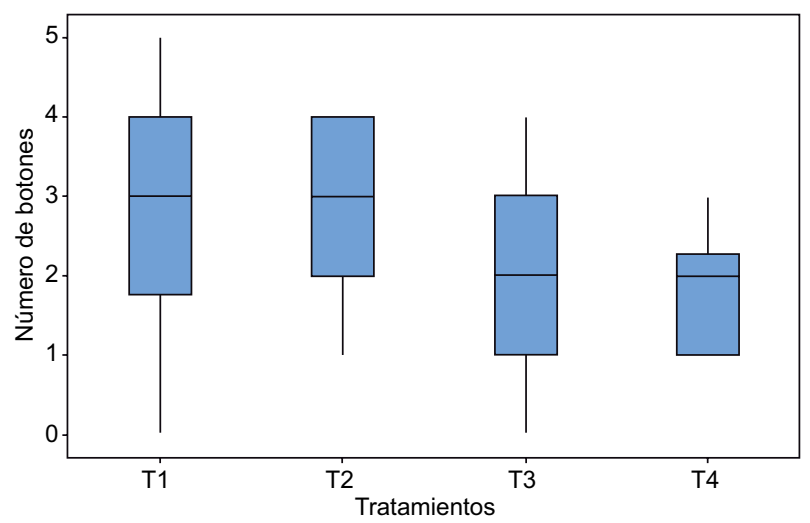

Fig. 1. Comparación de distribuciones de botones producidos por rosal según el tratamiento. $\mathrm{T}=$ tratamiento $($ Cuadro I). Se muestran los datos de los botones comprendidos entre el segundo y tercer cuartil, la línea horizontal es la mediana y las líneas verticales el rango de valores totales obtenidos

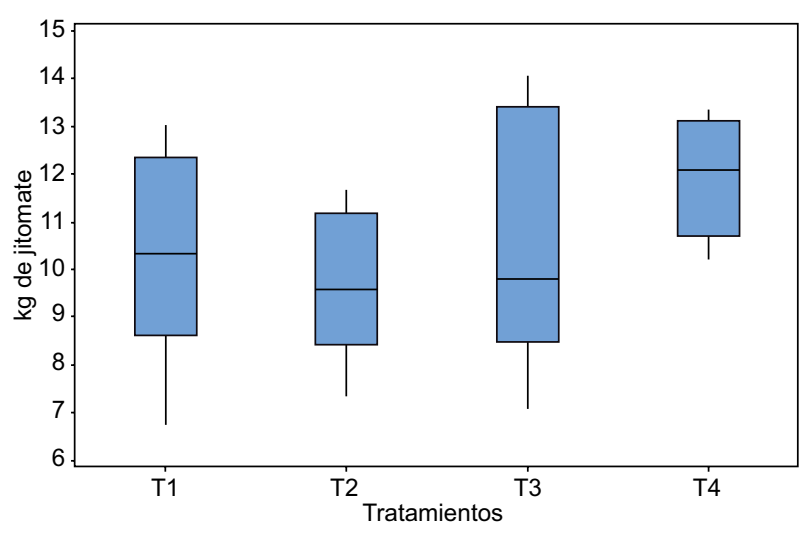

Fig. 2. Comparación de distribuciones de kg de jitomate producidos por planta según el tratamiento. $\mathrm{T}=$ tratamiento (Cuadro I). Se muestran los datos de los kg de jitomate comprendidos entre el segundo y tercer cuartil, la línea horizontal es la mediana y las líneas verticales el rango de valores totales obtenidos

Los resultados permiten suponer que el T4, correspondiente al sustrato biofísico formulado con $50 \%$ compost $+25 \%$ poliestireno $+25 \%$ tepetate puede sustituir a la tierra negra comúnmente empleada como sustrato en agricultura urbana en el cultivo de rosas sin aparentes afectaciones a la planta. Mientras que para el cultivo de jitomate, los 2 sustratos propuestos pueden sustituir al sustrato natural o al comercial.

Esta respuesta favorable de los sustratos propuestos puede deberse a la materia orgánica del compost aunado a la actividad microbiana que imparte al sustrato propiedades físicas, químicas y biológicas que se traducen en capacidad para amortiguar deficiencias nutricionales o fitotóxicas (Julca-Otiniano et al. 2006).

Estos resultados concuerdan con los hallazgos de Atiyeh et al. (2001), quienes sostienen que los efectos benéficos del compost sobre la productividad se generan cuando se utiliza en intervalos de $10 \%$ a $50 \%$. Así como lo demostrado por Lakhdar et al. (2008) quienes al adicionar compost al suelo, mitigaron el efecto negativo de la salinidad por $\mathrm{Na}$ y $\mathrm{Cl}$ sobre el crecimiento de las plantas. También hubo consistencia en lo reportado por García-Albarado et al. (2010) quienes observaron que incrementar la proporción de compost en el sustrato, aumenta la concentración de bicarbonatos (Cuadro III).

Por otro lado, adicionar agregados minerales como roca fosfórica o magnesita subsanaría las deficiencias de $\mathrm{P}$ y $\mathrm{Mg}$ en los sustratos propuestos, pues son necesarios niveles adecuados de estos para lograr óptimos rendimientos y calidad en los cultivos (Romero et al. 2000).

\section{CONCLUSIONES}

La mezcla de compost obtenido del tratamiento de la fracción orgánica de residuos sólidos urbanos con poliestireno recuperado de los residuos y tepetate, puede sustituir a los sustratos urbanos y agrícolas tradicionales empleados en cultivo de rosas y jitomate saladette sin fertilización adicional.

Valorizar subproductos recuperados de los residuos para elaborar sustratos, contribuye a mitigar el impacto ambiental derivado del confinamiento de residuos y a reducir la presión sobre recursos naturales empleados en agricultura urbana y protegida mediante el aprovechamiento de materiales que pueden emplearse como materia prima para elaborar sustratos alternos.

Por otra parte, el no haber requerido fertilización representa una mejora en el empleo de sustratos, pues implica ventajas económicas y ambientales que reducen costos y efectos contaminantes. Por lo que los resultados obtenidos contribuyen a la gestión integral de residuos.

En cuanto a futuras investigaciones, se sugiere monitorear el posible cambio de salinidad en los sustratos propuestos, ya que aunque se obtuvieron rendimientos adecuados, contienen niveles de iones por arriba de los intervalos óptimos. Se recomienda evaluar las variables de respuesta de los sustratos biofísicos propuestos al enriquecerlos con fuentes 
minerales de nutrientes para llevarlos a niveles óptimos, así como monitorear la pérdida de nutrientes en los mismos conforme pasan ciclos fenológicos para establecer recomendaciones de enriquecimiento nutricional.

\section{AGRADECIMIENTOS}

Este estudio se realizó gracias al apoyo prestado por la empresa Biosistemas Sustentables, S.A.P.I. de C.V. Los autores agradecen a Erika Soto Bautista y a Rafael Morales Morales por su colaboración en las tareas de invernadero y laboratorio

\section{REFERENCIAS}

Acevedo-Sandoval O. A., Ortiz-Hernández L. E., FloresRomán D., Velásquez-Rodríguez A. S. y Flores-Castro K. (2003). Caracterización física y química de horizontes endurecidos (tepetates) en suelos de origen volcánico del Estado de México. Agrociencia 37 (5), 435-449.

Acosta-Duran C. M., Gallardo C. S., Kämpf A. N. y Bezerra C. F. (2008). Materiales regionales utilizados en Latinoamérica para la preparación de sustratos. Investigación Agropecuaria 5 (2), 93-106.

Alcántar-González G., Trejo-Téllez L. I. y Gómez-Merino F. C. (2016). Nutrición de cultivos. $2^{\mathrm{a}}$ ed. Colegio de Postgraduados, Texcoco, México, 443 pp.

Ansorena-Miner J. (1994). Sustratos: propiedades y caracterización. Mundi-Prensa. Madrid, España, 172 pp.

Atiyeh R. M., Edwards C. A., Subler S. y Metzger J. D. (2001). Pig manure vermicompost as a component of a horticultural bedding plant medium: effects on physicochemical properties and plant growth. Bioresour. Technol. 78 (1), 11-20.

DOI: $10.1016 / \mathrm{S} 0960-8524(00) 00172-3$

Ayala-Sierra A. y Valdez-Aguilar L. A. (2008). El polvo de coco como sustrato alternativo para la obtención de plantas ornamentales para transplante. Rev. Chapingo Ser. Hortic. 14 (2), 161-167.

Bárcenas Abogado P., Tijerina Chávez L., Martínez Garza A., Becerril Román A. E., Larqué Saavedra A. y Colinas de León M. T. (2002). Respuesta de tres materiales del genero Hylocereus a la salinidad sulfatico-clorhídrica. Terra Latinoam. 20 (2), 123-127.

BOE (2010). Real Decreto 865/2010 del 2 de julio. Sobre sustratos de cultivo. Madrid, España. Boletín Oficial del Estado 170, 61831-61859.

Cabrera R. I. (1999). Propiedades, uso y manejo de sustratos de cultivo para la producción de plantas en maceta. Rev. Chapingo Ser. Hortic. 5 (1), 5-11.
CONAFOR y UACh. (2013). Línea base nacional de degradación de tierras y desertificación. Comisión Nacional Forestal y Universidad Autónoma Chapingo. Informe. Zapopan, México, 161 pp.

Cotler H., López C. A. y Martínez-Trinidad S. (2011). ¿Cuánto nos cuesta la erosión de suelos? Aproximación a una valoración económica de la pérdida de suelos agrícolas en México. Investigación ambiental ciencia y política pública 3 (2), 31-43.

Cruz Crespo E., Sandoval Villa M., Volke Haller V., Ordaz Chaparro V., Tirado Torres J. L. y Sánchez Escudero J. (2010). Generación de mezclas de sustratos mediante un programa de optimización utilizando variables físicas y químicas. Terra Latinoam. 28 (3), 219-229.

Cruz-Crespo E., Can-Chulim A., Sandoval-Villa M., Bugarín-Montoya R., Robles-Bermúdez A. y JuárezLópez P. (2013). Sustratos en la horticultura. Biociencias 2 (2), 17-26.

Cuenca G., Cáceres A., Oirdobro G., Hasmi Z. y Urdaneta C. (2007). Las micorrizas arbusculares como alternativa para una agricultura sustentable en áreas tropicales. Interciencia 32 (1), 23-29.

Díaz Franco A., Gálvez López D. y Ortiz Cháirez F. (2015). Bioinoculación y fertilización química reducida asociadas con el crecimiento de planta y productividad de sorgo. Rev. Int. Contam. Ambie. 31 (3), 245-252.

Dorner J. y Dec D. (2007). La permeabilidad del aire y conductividad hidráulica saturada como herramienta para la caracterización funcional de los poros del suelo. R. C. Suelo Nutr. Veg. 7 (2), 1-13. DOI: $10.4067 / \mathrm{S} 0718-27912007000200001$

García C. O., Alcántar G. G. Cabrera R. I., Gavi R. F. y Volke H. V. (2001). Evaluación de sustratos para la producción de Epipremnum aureum y Spathiphyllum wallisii cultivadas en maceta. Terra Latinoam. 19 (3), 249-258.

García-Albarado J .C., Trejo-Téllez L. I., VelásquezHernández M. A., Ruiz-Bello A. y Gómez-Merino F. C. (2010). Crecimiento de petunia en respuesta a diferentes proporciones de composta en sustrato. Rev. Chapingo Ser. Hortic. 16 (2), 107-113.

Ghosh S., Lockwood P., Hulugalle N., Daniel H., Kristiansen P. y Dodd K. (2010). Changes in properties of sodic Australian vertisols with application of organic waste products. Soil Sci. Soc. Am. J. 74 (1), 153-160.

Guevara-Escobar A., Barcenas-Huante G., SalazarMartínez F. R., González-Sosa E. y Suzán-Azpiri H. (2005). Alta densidad de siembra en la producción de maíz con irrigación por goteo subsuperficial. Agrociencia 39 (4), 431-439.

Gutiérrez Pulido H., Gutiérrez González P., Garibay López C. y Díaz Caldera L. (2014). Análisis multivariado 
y QFD como herramientas para escuchar la voz del cliente y mejorar la calidad del servicio. Ingeniare. Rev. Chil. Ing. 22 (1), 62-73. DOI: $10.4067 /$ S0718-33052014000100007

INECC y SEMARNAT (2012). Diagnóstico básico para la gestión de residuos. Centro Nacional de Investigación y Capacitación Ambiental. Instituto Nacional de Ecología y Cambio Climático - Secretaría de Medio Ambiente y Recursos Naturales. Informe. Ciudad de México, México, 201 pp.

Julca-Otiniano A., Meneses-Florián L., Blas-Sevillano R. y Bello-Amez S. (2006). La materia orgánica, importancia y experiencia de uso en la agricultura. Idesia 24 (1), 49-61.

DOI: $10.4067 / \mathrm{S} 0718-34292006000100009$

Lakhdar A., Hafsi C., Rabhi M., Debez A., Montemurro F., Abdelly C., Jedidi N. y Ouerghi Z. (2008). Application of municipal solid waste compost reduces the negative effects of saline water in Hordeum maritimum L. Bioresour. Technol. 99 (15), 7160-7167.

DOI: 10.1016/j.biortech.2007.12.071

Lemaire F., Dartigues A., Riviére L. M., Charpienter P. y Morel P. (2005). Cultivos en macetas y contenedores. Principios agronómicos y aplicaciones. Ediciones Mundi-prensa, Madrid, España, 232 pp.

Márquez-Hernández C., Cano-Ríos P., Chew-Madinaveitia Y. I., Moreno-Reséndez A. y Rodríguez-Dimas N. (2006). Sustratos en la producción orgánica de tomate cherry bajo invernadero. Rev. Chapingo Ser. Hortic. 12 (2), 183-189

Martínez V. E. y López F. (2001). Efecto de hidrocarburos en las propiedades físicas y químicas de suelo arcilloso. Terra Latinoam. 19 (1), 9-17.

McKean S. (1993). Manual de análisis de suelos y tejido vegetal una guía teórica y práctica de metodologías. Documento de trabajo 129 CIAT [en línea]. http:// ciat-library.ciat.cgiar.org/Articulos_Ciat/Digital/S593. M2_Manual_de_an\%C3\%A1lisis_de_suelos_y_tejido_vegetal_Una_gu $\% \mathrm{C} 3 \% \mathrm{ADa}$ _te $\% \mathrm{C} 3 \% \mathrm{~B} 3$ rica_y_ pr\%C3\%A1ctica_de_metodologia.pdf 09/02/2015.

Montgomery D. R. (2007). Soil erosion and agricultural sustainability. P. Natl. Acad. Sci. U. S. A. 104 (33), 13268-13272.

DOI: $10.1073 /$ pnas.0611508104

Moreno Reséndez A., Valdés Perezgasga M. T. y Zarate López T. (2005). Desarrollo de tomate en sustratos de vermicompost/arena bajo condiciones de invernadero. Agric. Téc. 65 (1), 26-34.

DOI: $10.4067 / \mathrm{S} 0365-28072005000100003$

Moreno Reséndez A., Aguilar Durón J. y Luévano González A. (2011). Características de la agricultura protegida y su entorno en México. Rev. Mex. Agron. 15 (29), 763-774.
Mujica Pérez Y. (2012). Inoculación de hongos micorrízicos arbusculares (HMA) por dos vías diferentes en el cultivo del tomate (Solanum lycopersicum L.). Cultrop. 33 (4), 71-76.

Olivares-Campos M. A., Hernández-Rodríguez A., Vences-Contreras C. Jáquez-Balderrama J. L. y OjedaBarrios D. (2012). Lombricomposta y composta de estiércol de ganado vacuno lechero como fertilizantes y mejoradores de suelo. Universidad y Ciencia 28 (1), 27-37.

Ortega-Martínez L. D., Ocampo-Mendoza J., SandovalCastro E., Martínez-Valenzuela C., Huerta-De La Peña A. y Jaramillo-Villanueva J. L. (2014). Caracterización y funcionalidad de invernaderos en Chignahuapan Puebla, México. Biociencias 2 (4), 261-270.

Paneque-Pérez V. M., Calaña-Naranjo M., Borges-Benítez Y., Hernández-García T. y Caruncho-Contreras M. (2010). Manual de técnicas analíticas para análisis de suelo, foliar, abonos orgánicos y fertilizantes químicos. $2^{\mathrm{a}}$ ed. Instituto Nacional de Ciencias Agrícolas (INCA). La Habana, Cuba, 160 pp.

Parra-Delgado J. M., Velázquez-Alcaraz T. J., QueroGutiérrez E., Partida-Ruvalcaba L., Díaz-Valdés T., Galván-Piña B. y Ayala-Tafoya F. (2014). Uso de composta, minerales primarios amorfos y microorganismos para la producción y calidad de tomate. Intropica 9, 102-110.

DOI: $10.21676 / 23897864.1437$

Raj D. y Antil R. S. (2011). Evaluation of maturity and stability parameters of composts prepared from agro-industrial wastes. Bioresour. Technol. 102 (3), 2868-2873.

DOI: $10.1016 /$ j.biortech.2010.10.077

Rodríguez-Fuentes H. y Rodríguez-Absi J. (2002). Métodos de análisis de suelos y plantas. $2^{\mathrm{a}}$ edición, Trillas, Ciudad de México, México, 239 pp.

Romero Lima M. R., Trinidad Santos A., García Espinosa R. y Ferrera Cerrato R. (2000). Producción de papa y biomasa microbiana en suelo con abonos orgánicos y minerales. Agrociencia 34 (3), 261-269.

Ruiz-Ramírez J. (2010). Eficiencia relativa y calidad de los experimentos de fertilización en el cultivo de caña de azúcar. Terra Latinoam. 28 (2), 149-154.

SAGARPA (2015). Atlas agroalimentario. Secretaría de Agricultura, Ganadería, Desarrollo Rural, Pesca y Alimentación. Atlas. Ciudad de México, México, $220 \mathrm{pp}$.

Saldaña Durán C. E., Hernández Rosales I. P., Messina Fernández S. y Pérez Pimienta J. A. (2013). Caracterización física de los residuos sólidos urbanos y el valor agregado de los materiales recuperables en el vertedero El Iztete, de Tepic-Nayarit, México. Rev. Int. Contam. Ambie. 29 (3), 25-32. 
SE (2014). Norma Mexicana NMX-AA-170-SCFI-2014. Certificación de la operación de viveros forestales. Secretaría de Economía. Diario Oficial de la Federación. 22 de septiembre de 2014.

SEMARNAT (2001). Norma Oficial Mexicana NOM021-SEMARNAT-2001. Fertilidad, salinidad y clasificación de suelos, estudio, muestreo y análisis. Secretaría de Medio Ambiente y Recursos Naturales. Diario Oficial de la Federación. 7 de diciembre de 2001.

SEMARNAT (2009). Informe de la situación del medio ambiente en México. Secretaría del Medio Ambiente y Recursos Naturales. Compendio de estadísticas ambientales 2008. Ciudad de México, México, 358 pp.

SEMARNAT (2013). Anuario estadístico de la producción forestal 2013. Secretaría de Medio Ambiente y Recursos Naturales. Informe. Ciudad de México, México, $236 \mathrm{pp}$.

SMA (2006). Norma Técnica Estatal Ambiental NTEA006-SMA-RS-2006. Que establece los requisitos para la producción de los mejoradores de suelo elaborados a partir de residuos orgánicos. Secretaría del Medio Ambiente. Gaceta del Gobierno del Estado de México. 9 de octubre de 2006

Urbano-López de Meneses B. (2013). Naturación urbana, un desafío a la urbanización. Rev. Chapingo Ser. Cienc. For. Ambient. 19 (2), 225-236.

DOI: 10.5154/r.chscfa.2013.01.004

Vargas-Tapia P., Castellanos-Ramos J. Z., Muñoz-Ramos J. J., Sánchez-García P., Tijerina-Chávez L., LópezRomero R. M., Martínez-Sánchez C. y OjodeaguaArredondo J. L. (2008). Efecto del tamaño de partícula sobre algunas propiedades físicas del tezontle de Guanajuato, México. Agric. Téc. Méx. 34 (3), 323-331.

Vence L. B. (2008). Disponibilidad de agua-aire en sustratos para plantas. Cienc. Suelo 26 (2), 105-114.

Zapata N., Guerrero F. y Polo A. (2005). Evaluación de corteza de pino y residuos urbanos como componentes de sustratos de cultivo. Agric. Téc. 65 (4), 378-387. DOI: $10.4067 / \mathrm{S} 0365-28072005000400004$ 